\title{
Biomedical Applications of Stereoscopy for Three-Dimensional Surface Reconstruction in Scanning Electron Microscopes
}

*Correspondence to:

Kim KW,

Tel: +82-54-530-1246

Fax: +82-54-530-1248

E-mail: kiwoo@knu.ac.kr

Received June 19, 2016

Revised June 25, 2016

Accepted June 25, 2016

\author{
Ki Woo Kim ${ }^{1,2, *}$ \\ ${ }^{1}$ School of Ecology and Environmental System, Kyungpook National University, Sangju 37224, Korea
2Tree Diagnostic Center, Kyungpook National University, Sangju 37224, Korea \\ ${ }^{1}$ School of Ecology and Environmental System, Kyungpook National University, Sangju 37224, Korea
2Tree Diagnostic Center, Kyungpook National University, Sangju 37224, Korea
}

The scanning electron microscope (SEM) offers two-dimensional (2D) micrographs of three-dimensional (3D) objects due to its inherent operating mechanisms. To overcome this limitation, other devices have been used for quantitative morphological analysis. Many efforts have been made on the applications of software-based approaches to 3D reconstruction and measurements by SEM. Based on the acquisition of two stereo images, a multi-view technique consists of two parts: (i) geometric calibration and (ii) image matching. Quantitative morphological parameters such as height and depth could be nondestructively measured by SEM combined with special software programs. It is also possible to obtain conventional surface parameters such as roughness and volume of biomedical specimens through 3D SEM surface reconstruction. There is growing evidence that conventional 2D SEM without special electron detectors can be transformed to 3D SEM for quantitative measurements in biomedical research.

Key Words: Scanning electron microscope, Stereo pairs, Three-dimension, Tilting

\section{BEYOND 2D SCANNING ELECTRON MICROSCOPES}

The scanning electron microscope (SEM) is commonly employed to observe biological objects with various dimensions, because of its large depth-of-focus, broad magnification range, and high spatial resolution down to $1 \mathrm{~nm}$ (Schroettner et al., 2006). Due to the inherent operating mechanisms of SEM, three-dimensional (3D) structures of biological objects in nature are projected onto a two-dimensional (2D) image plane in SEM (Kim, 2015). It is therefore impossible to take direct measurements of conventional morphological parameters such as the height and depth of 3D structures by conventional SEM (Kim, 2015). To overcome this limitation, other devices such as confocal laser scanning microscopes, atomic force microscopes, and surface profilers have been widely used for quantitative morphological analysis (Kim, 2006; Kim et al., 2011; Schroettner et al., 2006; Szczepanowska et al., 2014). There is a growing body of research on the applications of softwarebased approaches to 3D reconstruction and measurements (Andruleit et al., 2006; Scherer, 2002). Combined with SEM, the software solution to morphological analysis has improved the performance of SEM for imaging and analyzing biological objects (Limandri et al., 2016). It alleviates the additional devices, time, and labor taken for conventional specimen preparations for morphological analysis. Many disciplines of science ranging from biology to cultural heritage conservation science could benefit from 3D SEM surface reconstruction techniques (Tafti et al., 2015). This review aims to provide the techniques for acquiring stereo images, and highlight a few published examples.

\section{IMAGE ACQUISITION FOR STEREOSCOPY}

There has been much progress in developing 3D SEM surface reconstruction algorithms, which can be categorized into three classes: (i) single-view, (ii) multi-view, and (iii) hybrid

(a) This is an open-access article distributed under the terms of the Creative Commons Attribution Non-Commercial License (http://creativecommons.org/licenses/by-nc/4.0) which permits unrestricted noncommercial use, distribution, and reproduction in any medium, provided the original work is properly cited.

Copyrights @ 2016 by Korean Society of Microscopy 
(Fig. 1) (Tafti et al., 2015). One of the most popular methods for 3D reconstruction in SEM is a multiple-view approach, which is based on the acquisition of two stereo images (stereo

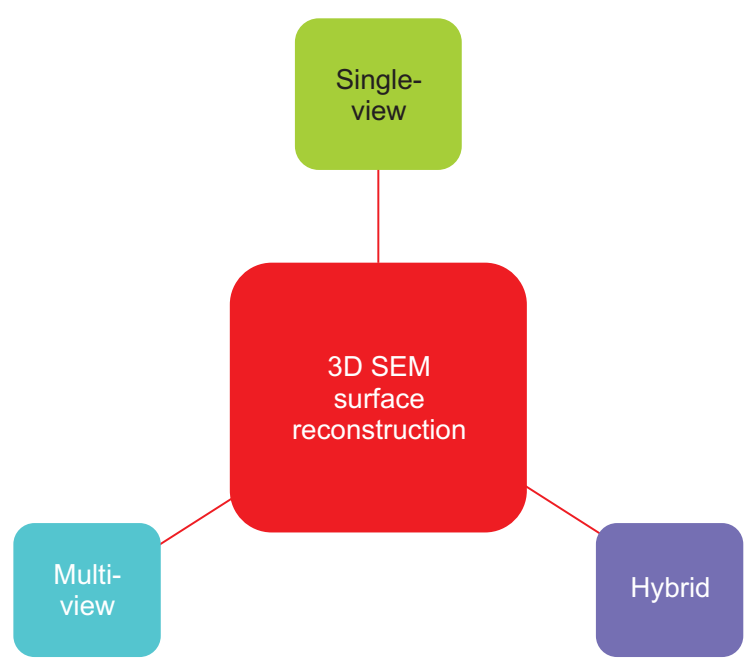

Fig. 1. Three major categories of three-dimensional scanning electron microscope (3D SEM) surface reconstruction algorithms. Modified from Tafti et al. (2015) (Micron 78, 54-66) with permission. pairs) (Limandri et al., 2016). The theoretical basis of the technique is dated back to Piazzesi (1973).

The multiple-view approach consists of two parts: (i) geometric calibration and (ii) image matching (Scherer, 2002). For geometric calibration, the two images are obtained under two distinct views using SEM equipped with a eucentric tilting stage (Scherer, 2002). The device parameters such as tilt angle, magnification, working distance, and image pixel size should be recorded and input for proper calibration. Surface points can be identified relative to a reference point, and metrically correct $3 \mathrm{D}$ points can be calculated and reconstructed using the geometric relationships such as the distances between them and their relative slopes (Bharadwaj et al., 2012; Limandri et al., 2016; Scherer, 2002; Tafti et al., 2015).

\section{MORPHOLOGICAL ANALYSIS OF PLANKTON}

Ocean plankton were collected and observed without conventional chemical preparations using a field emission SEM (Fig. 2A) (Andruleit et al., 2006). Two tilt images were acquired and further processed with software programs (XL pro [Soft Imaging System, Germany] and MeX [Alicona,
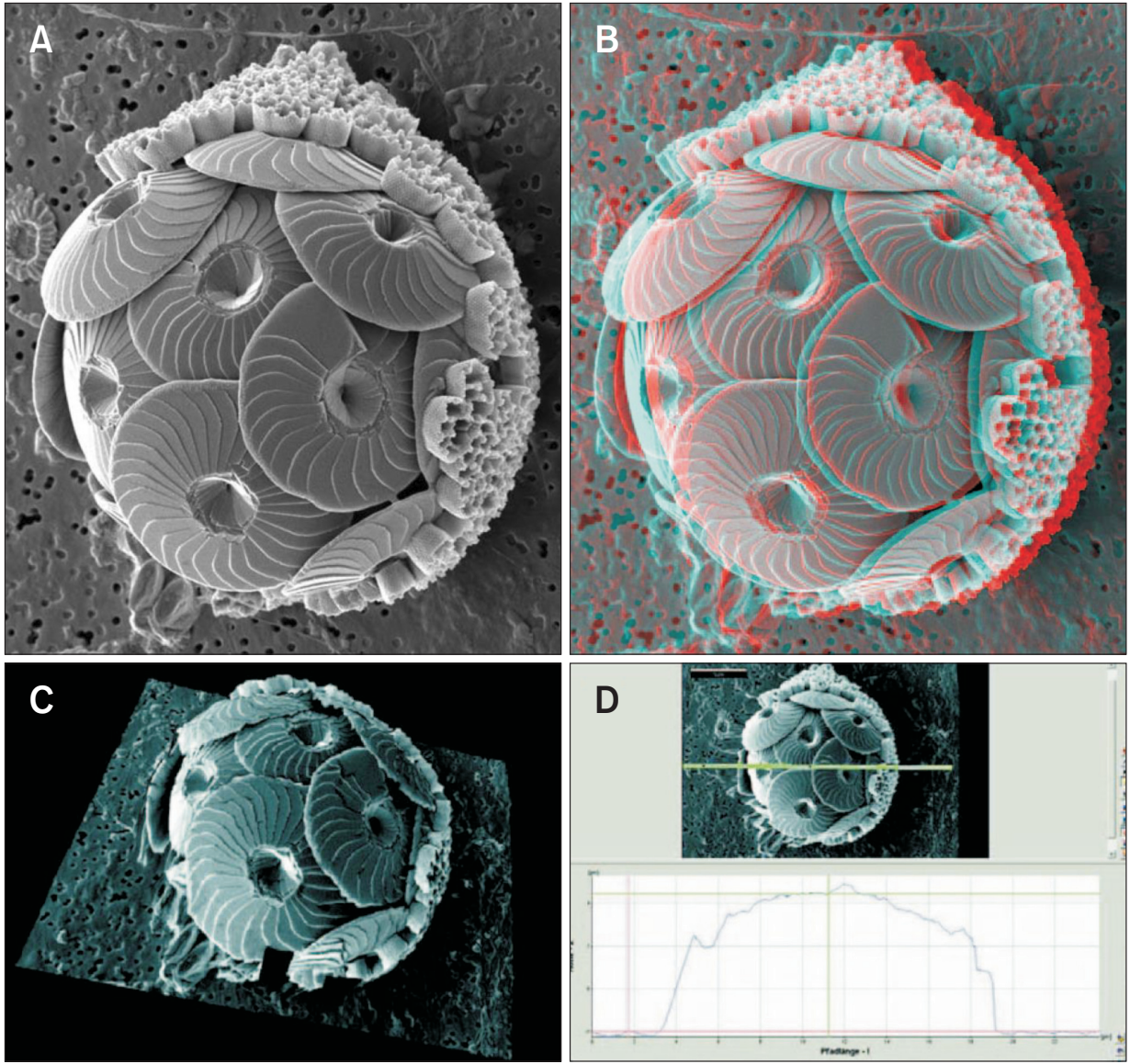

Fig. 2. An ocean plankter Calcidiscus quadriperforatus. (A) Field emission scanning electron micrograph. (B) Stereo image. The protrusion is evident with red-green glasses. (C) Three-dimensional surface reconstruct constructed from stereo images. (D) Digital elevation model. The angles between plates can be nondestructively measured. Reproduced from Andruleit et al. (2006) $(J$. Nannoplankton. Res. 28, 1-16) with permission from the publisher. 
Austria]) to produce stereo images (Fig. 2B). 3D surface reconstruction was performed to generate a digital elevation model of the species (Fig. 2C and D). The 3D imaging with stereoscopy will increase the understanding of functional morphology to elucidate the size-weight relationships of plankton (Andruleit et al., 2006).

\section{HEIGHT AND DEPTH MEASUREMENTS OF FUNGAL STRUCTURES}

It is common to observe the spores of rust fungi including Puccinia miscanthi on the leaf epidermis (Fig. 3A) (Kim,
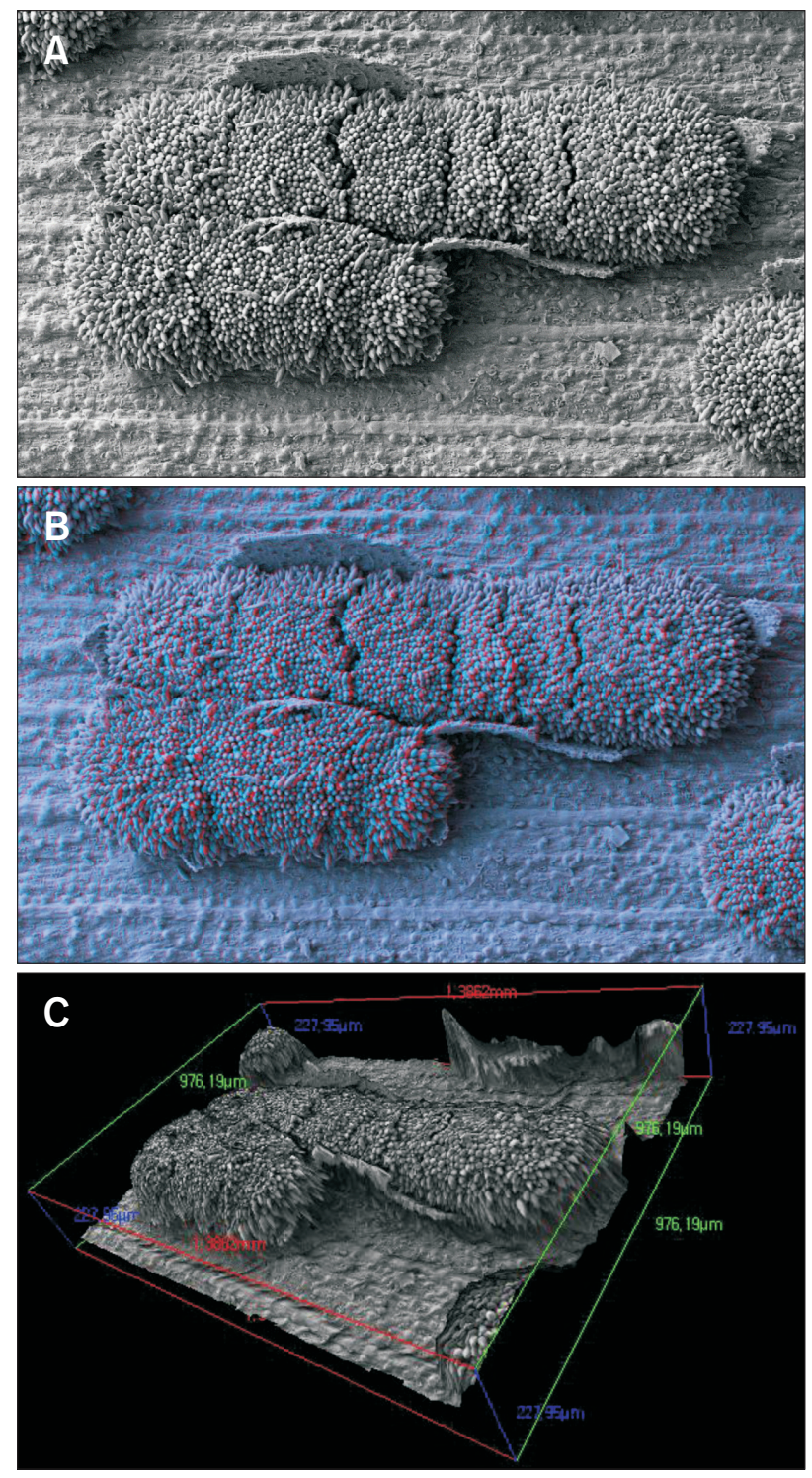

Fig. 3. A plant pathogenic fungus Puccinia miscanthi on the leaf surface of Miscanthus sinensis. (A) Field emission scanning electron micrograph. (B) Stereo image. (C) Three-dimensional surface reconstruct. The height of fungal structures from the leaf epidermis can be obtained. Reproduced from Kim (2015) (Micron 73, 15-20) with permission from the publisher.
2015). The fungal eruptions were more pronounced from stereo images (Fig. 3B) with red-green glasses than conventional micrographs, as described in Bozzola and Russell (1999). Without mechanical sectioning and special electron detectors, the height of the fungal eruptions could be nondestructively measured using the digital elevation model (Fig. 3C). Even the depth of fungal fruiting structures such as perithecia on pigmented paper could be also estimated by $3 \mathrm{D}$ SEM (Szczepanowska et al., 2014).

\section{ROUGHNESS ANALYSIS OF EXTRACELLULAR MATRIX FOR CANCER RESEARCH}

Type- 1 collagen scaffolds were made and inoculated with Escherichia coli strains (Bharadwaj et al., 2012). Roughness parameters were derived from the surface profiles of $3 \mathrm{D}$ reconstructs. Uninoculated scaffolds remained intact as viewed in 3D surface reconstructs (Fig. 4A). However, the inoculated scaffolds exhibited significant collagen degradation (Fig. 4B). The substrate degradation by the bacterial inoculation was also confirmed by surface roughness analysis (data not shown).
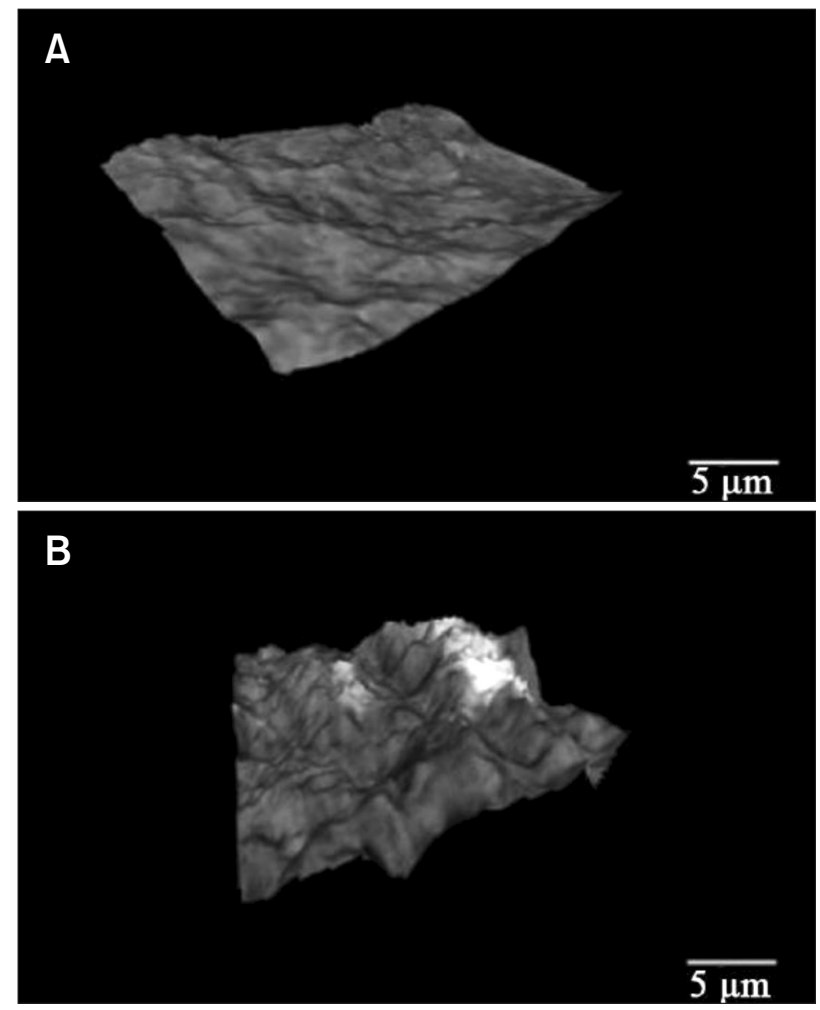

Fig. 4. Three-dimensional surface reconstructs of type-1 collagen scaffolds. (A) Uninoculated scaffold. It remained intact. (B) Escherichia coli-inoculated scaffold. It showed the substrate degradation by the bacterial inoculation. Reproduced from Bharadwaj et al. (2012) (J. Biomater. Nanobiotechnol. 3, 70-78) with permission from the publisher. 
A

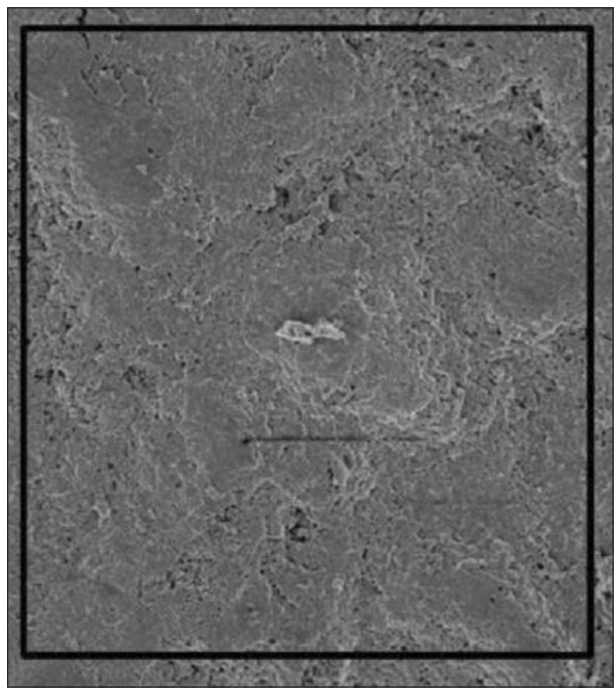

C

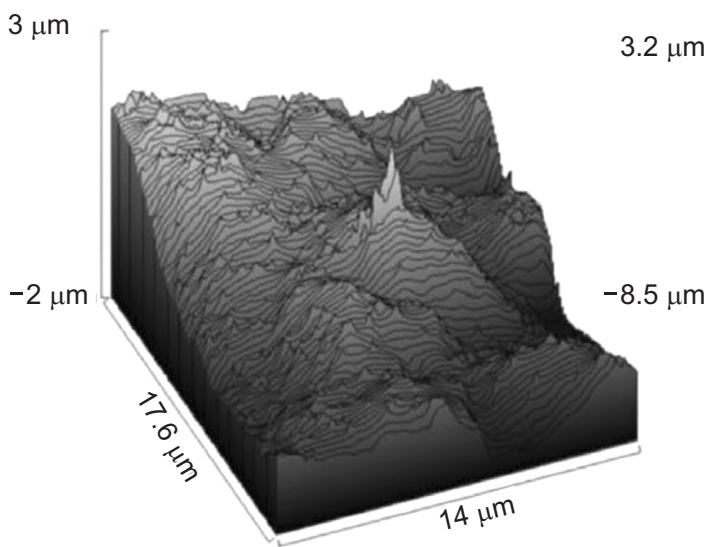

B

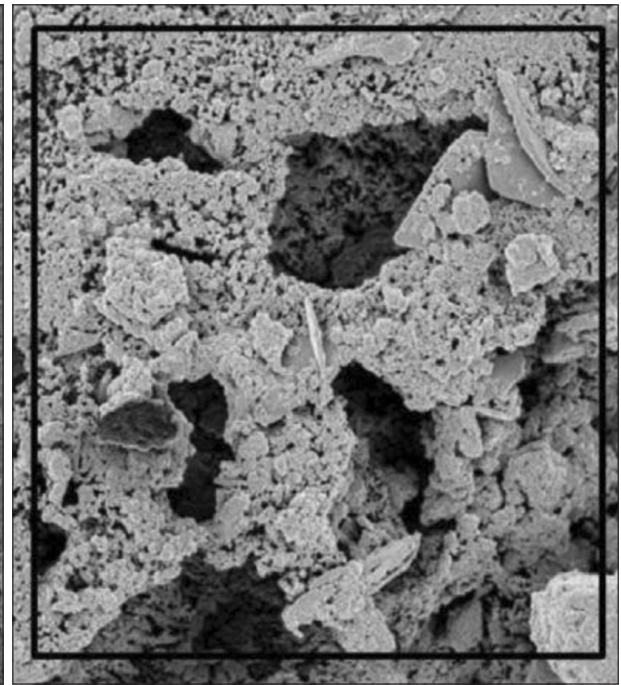

D

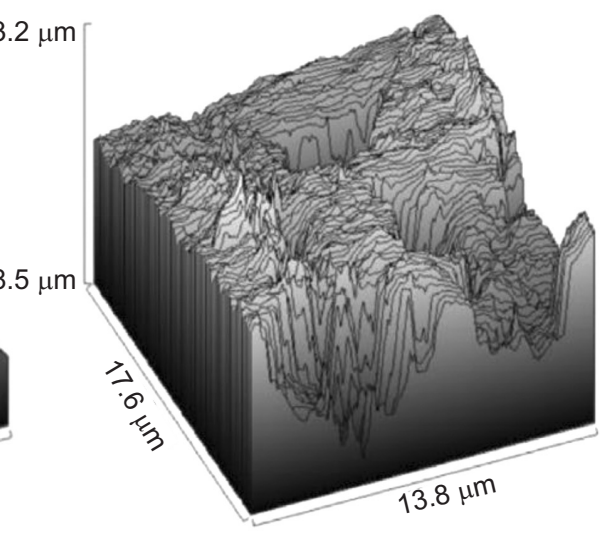

Fig. 5. Tooth surface with different types of dental treatments. (A, B) Scanning electron micrographs of teeth with different types of dental treatments. (C, D) Height maps of inlets of Fig. 5A and $\mathrm{B}$ from three-dimensional surface reconstructs, respectively. Reproduced from from Limandri et al. (2016) (Micron 84, 54-60) with permission from the publisher.

\section{SURFACE CHARACTERIZATION OF DENTAL ENAMEL}

Teeth (mainly third molars) of adult patients were extracted, subjected to an ultrasonic cleaning process, and sputter-coated with gold (Limandri et al., 2016). Stereo images were acquired with two tilt angles $\left(0^{\circ}\right.$ and $\left.5^{\circ}\right)$ and further processed for 3D reconstruction. SEM observations showed tooth surfaces with different whitening and cleaning treatments (Fig. 5A and B). Height maps derived from 3D surface reconstruction were used to characterize surface parameters caused by different dental treatments (Fig. 5C and D).

\section{CONCLUSIONS}

Conventional SEM provides 2D qualitative micrographs of 3D objects. Combined with special software programs, conventional 2D SEM can be transformed to 3D SEM for quantitative measurements. 3D SEM without special electron detectors can be employed in a variety of experiments ranging from morphological analysis of biological objects to the degradation of extracellular matrix in cancer research.

\section{CONFLICT OF INTEREST}

No potential conflict of interest relevant to this article was reported. 


\section{REFERENCES}

Andruleit H, Geisen M, Stäger S (2006) Stereo-microscopy of coccolithophores: modern applications for imaging and morphological analysis. J. Nannoplankton. Res. 28, 1-16.

Bharadwaj S, Nekrasov V, Vishnubhotla R, Foster C, and Glover S C (2012) Commensal $E$. coli strains uniquely alter the ECM topography independent of colonic epithelial cells. J. Biomater. Nanobiotechnol. 3, 70-78.

Bozzola J J and Russell L D (1999) Electron Microscopy: Principles and Techniques for Biologists, 2nd ed., (Jones and Bartlett Publishers, Boston).

Kim K W (2006) Atomic force microscopy for direct imaging and nanoscale morphometry of the rice false smut fungus Ustilaginoidea virens. J. Phytopathol. 154, 751-754.

Kim K W (2015) Three-dimensional surface reconstruction and in situ site-specific cutting of the teliospores of Puccinia miscanthi causing leaf rust of the biomass plant Miscanthus sinensis. Micron 73, 1520

Kim K W, Lee I J, Kim C S, Lee D K, and Park E W (2011) Micromorphology of epicuticular waxes and epistomatal chambers of pine species by electron microscopy and white light scanning interferometry. Microsc. Microanal. 17, 118-124.

Limandri S, Josa V G, Valentinuzzi M C, Chena M E, and Castellano G (2016) 3D scanning electron microscopy applied to surface characterization of fluorosed dental enamel. Micron 84, 54-60.

Piazzesi G (1973) Photogrammetry with the scanning electron microscope. J. Phys. E: Sci. Instrum. 6, 392-396.

Scherer S (2002) 3D surface analysis in scanning electron microscopy. Imag. Microsc. 3, 45-46.

Schroettner H, Schmied M, and Scherer S (2006) Comparison of 3D surface reconstruction data from certified depth standards obtained by SEM and an Infinite Focus Measurement Machine (IFM). Microchim. Acta 155, 279-284.

Szczepanowska H, Mathia T G, and Belin P (2014) Morphology of fungal stains on paper characterized with multi-scale and multi-sensory surface metrology. Scanning 36, 76-85.

Tafti A P, Kirkpatrick A B, Alavi Z, Owen H A, and Yu Z (2015) Recent advances in 3D SEM surface reconstruction. Micron 78, 54-66. 